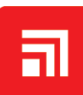 CHITKARA \\ Journal of Nuclear Physics, Material Sciences, Radiation and Applications

\section{On The Role of Nuclear Quantum Gravity In Understanding Nuclear Stability Range of $Z=2$ to 118}

\author{
U. V. S. Seshavatharam ${ }^{1 *}$ and S. Lakshminarayana ${ }^{2}$ \\ ${ }^{1}$ Honorary faculty, I-SERVE, Survey no-42, Hitech city, Hyderabad-84,Telangana, India \\ ${ }^{2}$ Department of Nuclear Physics, Andhra University, Visakhapatnam-03, Andhra Pradesh, India
}

*Email: seshavatharam.uvs@gmail.com

\section{ARTICLE INFORMATION}

Received: October 17, 2019

Revised: January 30, 2020

Accepted: February 18, 2020

Published online: February 18, 2020

\section{Keywords:}

Four gravitational constants, Compound reduced Planck's constant, Nuclear elementary charge, Strong coupling constant, Nuclear binding energy, Nuclear stability limits, Super heavy elements

DOI: $10.15415 /$ jnp.2019.71005

\section{ABSTRACT}

To understand the mystery of final unification, in our earlier publications, we proposed two bold concepts: 1) There exist three atomic gravitational constants associated with electroweak, strong and electromagnetic interactions. 2) There exists a strong elementary charge in such a way that its squared ratio with normal elementary charge is close to reciprocal of the strong coupling constant. In this paper we propose that, $\hbar c$ can be considered as a compound physical constant associated with proton mass, electron mass and the three atomic gravitational constants. With these ideas, an attempt is made to understand nuclear stability and binding energy. In this new approach, with reference to our earlier introduced coefficients $\mathrm{k}=0.00642$ and $\mathrm{f}=0.00189$, nuclear binding energy can be fitted with four simple terms having one unique energy coefficient. The two coefficients can be addressed with powers of the strong coupling constant. Classifying nucleons as 'free nucleons' and 'active nucleons', nuclear binding energy and stability can be understood. Starting from, number of isotopes seems to increase from 2 to 16 at and then decreases to 1 at For $Z>=84$, lower stability seems to be, $A_{\text {lower }}=(2.5$ to 2.531)Z.

\section{Introduction to Large Gravitational Coupling Constants}

To understand the strong interaction, from 1974 to 1993, Tennakone, De Sabbata, Gasperini, Abdus Salam, Sivaram and K.P. Sinha [1-4] tried to introduce a large nuclear gravitational coupling constant. To understand weak interactions, in 2013, Roberto Onofrio [5] introduced a large electroweak gravitational coupling constant. In our 2011 and 2012 papers [6, 7] and recent papers [8-20], we introduced a very large electromagnetic gravitational coupling constant. In this context, we appeal that,

1) Success of any unified model depends on its ability to involve gravity in microscopic models.

2) Full-fledged implementation of gravity in microscopic physics must be able to:
a) Estimate the ground state elementary particle rest masses of the three atomic interactions.
b) Estimate the coupling constants of the three atomic interactions.
c) Estimate the range of all interactions.
d) Estimate the Newtonian gravitational constant.

3) As the root is unclear and unknown, to make it success or to have a full-fledged implementation, one may be forced to consider a new path that may be out-of-scope of the currently believed unsuccessful unified physics.

4) In our approach,

a) We assign a different gravitational constant for each basic interaction.

b) We consider proton and electron as the two characteristic building blocks of the four basic interactions.

c) Finally, by eliminating the three atomic gravitational constants, we develop a characteristic relation for estimating the Newtonian gravitational constant.

d) During this journey, without considering arbitrary numbers or coefficients, we come across many strange and interesting relations for estimating other atomic and nuclear coupling constants.

5) We strongly believe that, with further study, research and synthesizing the noticed relations in a systematic approach, actual essence of final unification can be understood. 


\section{History and Current Status of Nuclear Binding Energy Scheme}

With respect to nuclear binding energy and semi empirical mass formula (SEMF), the inverse problem framework [21], allows to infer the underlying model parameters from experimental observation, rather than to predict the observations from the model parameters. Recently, the ground-state properties of nuclei with $\mathrm{Z}=8$ to 120 from the proton drip line to the neutron drip line have been investigated using the spherical relativistic continuum Hartree-Bogoliubov (RCHB) theory [22] with the relativistic density functional PC-PK1. In this context, in our recently published paper [8], we emphasized the fact that, physics and mathematics associated with fixing of the energy coefficients of SEMF are neither connected with residual strong nuclear force nor connected with strong coupling constant. N. Ghahramany and team members are constantly working on exploring the secrets of nuclear binding energy and magic numbers in terms of quarks [23, 24]. Very interesting point of their study is that - nuclear binding energy can be understood with two or three terms having single energy coefficient of the order of $10 \mathrm{MeV}$.

\section{Three Bold Ideas}

Even though celestial objects that show gravity are confirmed to be made up of so many atoms, so far scientists could not find any relation in between gravity and the atomic interactions. It clearly indicates that, there is something wrong in our notion of understanding or developing the unified physical concepts. To develop new and workable ideas, we emphasize that,

1) Whether particle's massive nature is due to electromagnetism or gravity or weak interaction or strong interaction or cosmic dust or something else, is unclear.

2) Without understanding the massive nature, it is not reasonable to classify the field created by any elementary particle.

3) All the four interactions seem to be associated with ( $\hbar c)$.

4) Nobody knows the mystery of ( $\hbar c)$ which seems to be a basic measure of angular momentum.

5) Nobody knows the mystery of existence, stability and behavior of 'proton' or 'electron'.

6) 'Mass' is a basic property of space-time curvature and basic ingredient of angular momentum.

7) Atoms are mainly characterized by protons and electrons.

8) 'Free neutron' is an unstable particle.

Based on the above points, we propose the following new and workable concepts.
Bold idea-1: The four basic interactions can be allowed to have four different gravitational constants.

Bold idea-2: There exists a strong elementary charge in such a way that its squared ratio with normal elementary charge is close to inverse of the strong coupling constant.

Bold idea-3: $(\hbar c)$ can be considered as a compound physical constant associated with proton mass, electron mass and the three atomic gravitational constants.

With the proposed first two [8-18] concepts, it seems possible to have many applications out of which nuclear stability and binding energy can be understood very easily. In addition to that, Newtonian gravitational constant can be estimated in a verifiable approach $[18,19,20]$. We appeal that, by considering the third bold idea, it may be possible to understand the combined role of the four gravitational constants in understanding the vector and tensor nature of fundamental forces and their interaction range.

\section{Quantitative Relations}

(1) Let, Electromagnetic gravitational constant $=$

$$
G_{e} \cong 2.374335 \times 10^{37} \mathrm{~m}^{3} \mathrm{~kg}^{-1} \mathrm{sec}^{-2}
$$

Nuclear gravitational constant $=$ $G_{s} \cong 3.329561 \times 10^{28} \mathrm{~m}^{3} \mathrm{~kg}^{-1} \mathrm{sec}^{-2}$

Weak gravitational constant $=$ $G_{w} \cong 2.909745 \times 10^{22} \mathrm{~m}^{3} \mathrm{~kg}^{-1} \mathrm{sec}^{-2}$

(2) $(\hbar c)$ can be considered as a compound physical constant,

$$
\begin{aligned}
\hbar c & \cong\left(\frac{G_{w}}{G_{s}}\right) G_{e} m_{p} m_{e} \\
& \cong \sqrt{\left(G_{s} m_{p} m_{e}\right)\left(G_{e} m_{e}^{2}\right)} \cong \frac{\left(G_{e}^{2} G_{N}\right)^{1 / 3} m_{p}^{4}}{m_{e}^{2}}
\end{aligned}
$$

where $G_{N}=$ Newtonian Gravitational constant.

(3) There exists a strong elementary charge $(e)$ in such a way that,

$$
\begin{aligned}
& \frac{m_{p}}{m_{e}} \cong\left(\frac{G_{s} m_{p}^{2}}{\hbar c}\right)\left(\frac{G_{e} m_{e}^{2}}{\hbar c}\right) \\
\cong & \left.\left(\frac{e_{s}^{2}}{4 \pi \varepsilon_{0} G_{s} m_{p}^{2}}\right) /\left(\frac{e^{2}}{4 \pi \varepsilon_{0} G_{e} m_{e}^{2}}\right)\right\} \\
\rightarrow & \left\{\begin{array}{l}
\frac{e_{s}^{2}}{e^{2}} \cong\left(\frac{G_{s} m_{p}^{3}}{G_{e} m_{e}^{3}}\right) \cong\left(\frac{G_{s} m_{p}^{2}}{\hbar c}\right)^{2} \cong \frac{1}{\alpha_{s}} \\
\frac{e_{s}}{e} \cong \sqrt{\frac{G_{s} m_{p}^{3}}{G_{e} m_{e}^{3}}} \cong\left(\frac{G_{s} m_{p}^{2}}{\hbar c}\right) \cong \sqrt{\frac{1}{\alpha_{s}}}
\end{array}\right\}
\end{aligned}
$$


Based on these relations,

$$
e_{s} \cong 2.9463591 e, \alpha_{s} \cong 0.1151937 \text { and } \frac{1}{\alpha_{s}} \cong 8.681032
$$

\section{Understanding Proton-neutron Mean Stability with Three Atomic Gravitational Constants}

In our recently published paper [8], we proposed the following semi empirical relations (4) to (7) for fitting nuclear stability and binding energy.

$$
\begin{aligned}
& s \cong\left\{\left(\frac{e_{s}}{m_{p}}\right) \div\left(\frac{e}{m_{e}}\right)\right\} \cong 0.001605 \\
&\left.\cong \sqrt{\frac{G_{s} m_{p}}{G_{e} m_{e}} \cong \frac{G_{s} m_{p} m_{e}}{\hbar c} \cong \frac{\hbar c}{G_{e} m_{e}^{2}} \cong \frac{G_{s}^{2}}{G_{e} G_{w}} \cong \frac{m_{p}}{M_{w}}}\right\} \\
& \text { where, } M_{w} \cong \sqrt{\hbar c / G_{w}} \cong 584.725 \mathrm{GeV} / c^{2}
\end{aligned}
$$

Nuclear beta stability line can be explained with a relation of the form,

$$
\begin{aligned}
& A_{s} \cong Z+N_{s} \cong 2 Z+s(2 Z)^{2} \cong 2 Z+(4 s) Z^{2} \\
& \cong 2 Z+k Z^{2} \cong Z(2+k Z)
\end{aligned}
$$

where $k \cong 4 s \cong 0.0064185$

Here we would like to appeal that, estimated $A_{S}$ can be considered as the mean stable mass number of $Z$. Here it is interesting to note that, in literature $[25,26]$, there exists a relation of the form, $N-Z \cong 0.006 A^{5 / 3}$.

\section{Understanding Nuclear Binding Energy}

For $(Z \approx 3$ to 118$)$, close to beta stability line, nuclear binding energy can be fitted with,

$$
B_{A_{s}} \cong\left\{\left(1-0.00189 \sqrt{Z N_{s}}\right) A_{s}-A_{s}^{1 / 3}-\left(\frac{Z}{N_{s}}\right)\right\} B_{0}
$$

See Figure 1. Dashed red curve plotted with relations (5) and (6) can be compared with the green curve plotted with the standard SEMF. For light, medium and heavy atomic nuclides, fit is reasonable.

Based on the proposed relations (4), (5) and (6) and with reference to Figure-1, we propose that,

(1) Nuclear unit radius can be expressed as,

$$
\begin{aligned}
& R_{0} \cong \frac{2 G_{s} m_{p}}{c^{2}} \cong 1.239291 \mathrm{fm} \\
& B_{0} \cong \frac{e_{s}^{2}}{4 \pi \varepsilon_{0} R_{0}} \cong \frac{e_{s}^{2}}{8 \pi \varepsilon_{0}\left(G_{s} m_{p} / c^{2}\right)} \cong 10.09 \mathrm{MeV}
\end{aligned}
$$

can be considered as the unique binding energy coefficient. With reference to the recommended [27] up quark rest energy of $2.15 \mathrm{MeV}$ and down quark rest energy of 4.7 $\mathrm{MeV}$, it is quite interesting to note that, $\frac{\left[\left(2 m_{u}+m_{d}\right) c^{2}+\left(m_{u}+2 m_{d}\right) c^{2}\right]}{2} \cong 10.275 \mathrm{MeV}$.

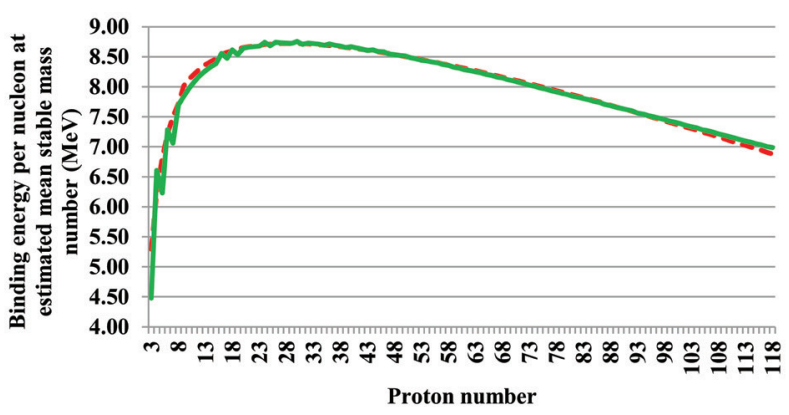

Figure 1: Binding energy per nucleon at estimated mean stable mass numbers of $\mathrm{Z}=3$ to 118 .

(3) For increasing $(Z, A)$, all nucleons will not involve in nuclear binding energy scheme.

(4) Nucleons that are not involving in nuclear binding energy scheme can be called as 'free nucleons' and can be represented by $A_{f} \cong k_{f} A \sqrt{Z N}$ where the coefficient $k_{f} \cong 0.00189$ can be called as 'Free nucleon number coefficient'. With reference to the semi empirical mass formula, quantitatively, $k_{f} \cong 2\left(a_{c} / a_{a}\right)^{2} \cong 0.0018753$ where $a_{c}=0.71 \mathrm{MeV}$ and $a_{a}=23.21 \mathrm{MeV}$.

(5) Nucleons that involve in nuclear binding energy scheme can be called as 'active nucleons' and can be represented by $A_{a} \cong A-A_{f} \cong A(1-0.00189 \sqrt{Z N})$.

(6) For $Z=11$ to 84 when $\left(A_{a}-2 Z\right) \cong 0$, corresponding A seems to represent the possible existence of lower stability line.

(7) The ad-hoc coefficient 0.00189 somehow, seems to lie between $\{s \cong 0.0016$ and $k \cong 0.0064\}$. With reference to electromagnetic interaction, we consider, $[k / \ln (30)] \cong 0.00189$ where 30 is a characteristic representation of atomic number below which strength of nuclear binding $[Z / 30]^{\sqrt{k}}\left(1 / \alpha_{s}\right) \cong[Z / 30]^{0.08} \times 8.68$. From $Z=30$ onwards, strength of nuclear binding energy remains constant at $\left(1 / \alpha_{s}\right) \cong 8.68$.

(8) Binding energy can be assumed to decrease with increasing radius.

(9) Decreasing proton-neutron ratio seems to play an interesting role in increasing binding energy.

(10) Considering isotopes, stable mass number plays an interesting role in estimating the binding energy of other stable and unstable isotopes in the form of $\left(\left(A_{s}-A\right)^{2} / A_{s}\right)$. It needs further investigation. 
Above and below the stable mass numbers, binding energy can be approximated with,

$$
B_{A} \cong\left\{(1-0.00189 \sqrt{Z N}) A-A^{1 / 3}-\left(\frac{Z}{N}\right)-\frac{\left(A_{s}-A\right)^{2}}{A_{s}}\right\} B_{0}
$$

See Figure 2 for the estimated isotopic binding energy of $\mathrm{Z}=50$. Dotted blue curve plotted with relations (5) and (7) can be compared with the green curve plotted with SEMF.

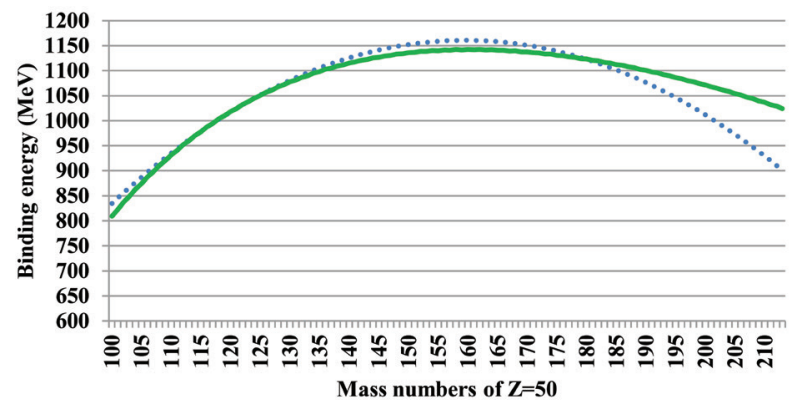

Figure 2: Isotopic binding energy of $Z=50$.

a) Based on Figures 1 and 2, it is possible to say that, Relations (5), (6) and (7) can also be given some priority in understanding nuclear binding energy scheme.

b) Estimated binding energy can also be compared with spherical relativistic continuum Hartree-Bogoliubov (RCHB) theory data [22] and Thomas-Fermi model (Table of nuclear masses, nsdssd.lbl.gov, 1994).

c) For $(N<Z)$ and $(N \approx Z)$ estimated binding energy seems to be increasing compared to SEMF estimation.

d) For $\left(A>>A_{s}\right)$, estimated binding energy seems to be decreasing compared to SEMF estimation.

\section{Discussion on the Proposed Nuclear Binding Energy Scheme}

(1) Nuclear binding energy can be understood with a single and unified energy coefficient.

(2) The new numbers ( $s$ and $k$ ) seem to play an interesting role in understanding nuclear stability and binding energy.

(3) With reference to stable mass number and similar to the famous relation $Z \cong A /\left(1.98+0.0153 A^{2 / 3}\right)$, proton number can also be estimated with [11], $Z \cong \frac{A}{1+\sqrt{1+k A}} \cong \frac{\sqrt{k A+1}-1}{k}$.

(4) Considering aterm of the form $\left(1-0.00189 \sqrt{Z \sqrt{N N_{s}}}\right)$ or by modifying the terms, $(Z / N)$ and $\left(\left(A_{s}-A\right)^{2} / A_{s}\right)$ binding energy for $\left(A<<A_{s}\right)$ and $\left(A>>A_{s}\right)$ can be understood.

(5) $\mathrm{Z}=(2$ to 118$)$, close to stable mass numbers, binding energy [8] can also be approximated with,

For $\mathrm{Z}<30$ and $A_{s} \cong Z(2+k Z)$,

$$
\left(B_{A_{s}}\right) \cong\left(\frac{Z}{30}\right)^{0.08}\left\{A_{s}-\left[\left(0.00189 N_{s}^{2}\right)+\frac{1}{2}\right]\right\} 9.16 \mathrm{MeV}
$$

For $\mathrm{Z} \geq 30$ and $A_{s} \cong Z(2+k Z)$,

$$
\left(B_{A_{s}}\right) \cong\left\{A_{s}-\left[\left(0.00189 N_{s}^{2}\right)+\frac{1}{2}\right]\right\} 9.16 \mathrm{MeV}
$$

$$
\text { where, }\left\{\begin{array}{l}
\frac{e_{s}^{2}}{8 \pi \varepsilon_{0}\left(G_{s} m_{p} / c^{2}\right)}-\left(\frac{e^{2}}{4 \pi \varepsilon_{0} R_{0}}\right) \cong 8.928 \mathrm{MeV} \\
\frac{e_{s}^{2}}{8 \pi \varepsilon_{0}\left(G_{s} m_{p} / c^{2}\right)}-\frac{3}{5}\left(\frac{e^{2}}{4 \pi \varepsilon_{0} R_{0}}\right) \cong 9.395 \mathrm{MeV} \\
\text { and } \frac{8.928+9.395}{2} \cong 9.16 \mathrm{MeV}
\end{array}\right\}
$$

See Figure 3. Dotted red curve plotted with relations (17) to (22) can be compared with the green curve plotted with the standard semi empirical mass formula (SEMF). For medium and heavy atomic nuclides, it is excellent. It seems that some correction is required for light and super heavy atoms.

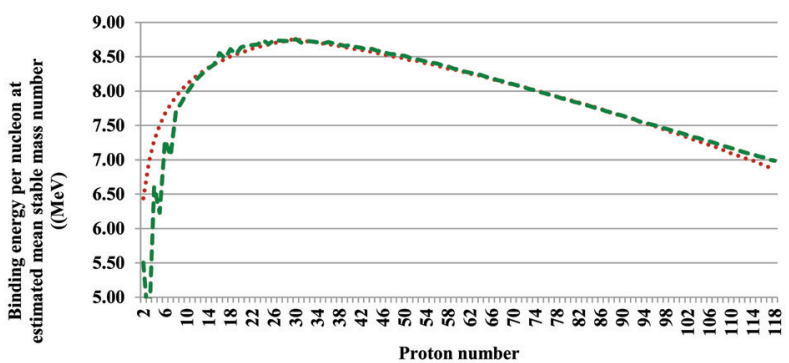

Figure 3: Binding energy per nucleon at estimated mean stable mass numbers of $\mathrm{Z}=2$ to 118 .

(6) In case of Deuteron, there exists no strong interaction between proton and neutron $[12,13]$.

\section{Understanding nuclear stability range}

\section{A) Basic observations and inferences}

Based on the above points we noticed that, lower and upper stability lines of $\mathrm{Z}$ can be fitted with three relations. Back 
ground physics of the three relations can be understood with the following points.

1) Active nucleon number seems to play an interesting role in estimating lower stability line of lower $\mathrm{Z}$ and higher stability line of higher $Z$. The corresponding relation seems to be, $\left(A_{a}-2 Z\right) \cong 0$. Proposed relations (9) and (13) seem to be appropriate numerical solutions.

2) Condition for higher stability line of lower $Z$ and lower stability line of higher $Z$ seems to be $(A-2 Z) \cong \frac{Z}{2}$ and $\frac{A}{Z} \cong 2.5 \cong\left(\frac{m_{n}-m_{p}}{m_{e}}\right) \cong 2 \cdot 531$. to be explored with further study.

3) At $\mathrm{Z}=83$ or 84 , nucleon-proton ratio seems to approach, $\frac{A}{Z} \cong 2.5$ (or) 2.531

4) As $Z$ is increasing from 2 to 52 , estimated isotopes number seems to increase from 2 to 16 respectively.

5) As $\mathrm{Z}$ is increasing from 53 to 84 , estimated isotopes number seems to decrease from 16 to 1 respectively.

6) As $\mathrm{Z}$ is increasing from 84 to 118 , estimated isotopes number seems to increase from 1 to 42 respectively.

7) At $Z=84$, estimated $\left(A_{S}\right)_{l_{\text {lower }}}\left(A_{S}\right)_{\text {mean }}$ and $\left(A_{S}\right)_{\text {upper }}$ seems to be equal.

8) $Z=84$ seems to be a transition point for changeover of lower and upper stability lines. Clearly speaking,

a) Lower stability line of $Z<84$, seems to become upper stability line of $Z>84$.

b) Upper stability line of $Z<84$, seems to become lower stability line of $Z>84$

9) Best possible range of stable super heavy elements [28, $29,30,31]$ can be estimated with relations (10) and (13). See Table -1 for a possible comparison of long lived super heavy elements.

10) Considering even-odd corrections, accuracy can be improved.

Table 1: Comparison of long lived super heavy elements.

\begin{tabular}{llll}
\hline $\begin{array}{l}\text { Proton } \\
\text { number }\end{array}$ & $\begin{array}{l}\text { Long lived } \\
\text { mass number }\end{array}$ & $\begin{array}{l}\text { Estimated } \\
\text { lower stable } \\
\text { mass number }\end{array}$ & $\begin{array}{l}\text { Estimated } \\
\text { mean stable } \\
\text { mass number }\end{array}$ \\
\hline 100 & 257 & 253 & 264 \\
101 & 258 & 256 & 267 \\
102 & 259 & 258 & 271 \\
103 & 266 & 261 & 274 \\
104 & 267 & 263 & 277 \\
105 & 268 & 266 & 281 \\
106 & 269 & 268 & 284 \\
107 & 270 & 271 & 288 \\
108 & 269 & 273 & 291 \\
109 & 278 & 276 & 294 \\
110 & 281 & 278 & 298 \\
111 & 282 & 281 & 301
\end{tabular}

\begin{tabular}{llll}
112 & 285 & 283 & 305 \\
113 & 286 & 286 & 308 \\
114 & 289 & 289 & 311 \\
115 & 290 & 291 & 315 \\
116 & 293 & 294 & 318 \\
117 & 294 & 296 & 322 \\
118 & 294 & 299 & 325 \\
\hline
\end{tabular}

\section{B) Relations connected with lower, mean and upper stability lines of $Z=2$ to 118}

\section{a) Lower stability line}

$$
\text { For, } Z \leq 84 \text {, }
$$$$
\left(A_{s}\right)_{\text {lower }} \cong 2 Z+\left(3.4 \times\left(\frac{Z}{30}\right)^{2.5}\right)
$$

where $\ln (30) \cong 3.4$

For, $Z>84$,

$$
\begin{aligned}
& \left(A_{s}\right)_{\text {lower }} \cong Z * 2.531 \\
& \text { where }\left(\frac{m_{n}-m_{p}}{m_{e}}\right) \cong 2.531
\end{aligned}
$$

b) Mean stability line

$$
\begin{aligned}
& \left(A_{s}\right)_{\text {mean }} \cong 2 Z+k Z^{2} \\
& \text { where } k \cong 0.0064185
\end{aligned}
$$

\section{c) Upper stability line}

$$
\begin{aligned}
& \text { For, } Z \leq 84, \\
& \left(A_{s}\right)_{\text {upper }} \cong Z * 2.531
\end{aligned}
$$

For, $Z>84$,

$$
\left(A_{s}\right)_{\text {upper }} \cong 2 Z+\left(3.4 \times\left(\frac{Z}{30}\right)^{2.5}\right)
$$

See the following Figures 4 and 5 and Table 2 .

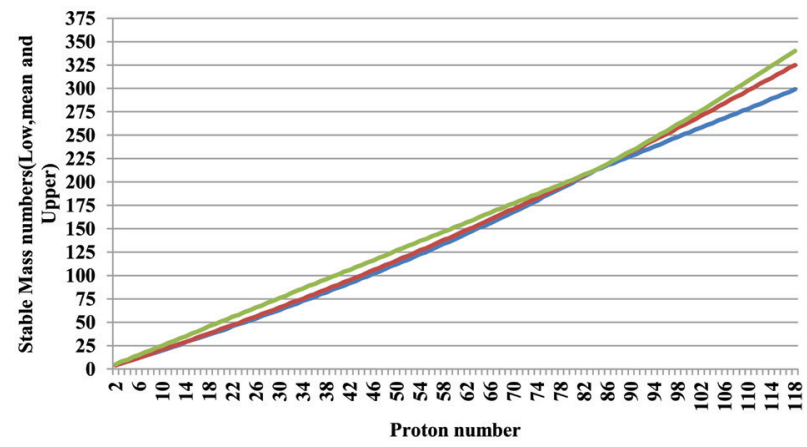

Figure 4: Estimated lower, mean and upper stable mass numbers of $\mathrm{Z}$. 


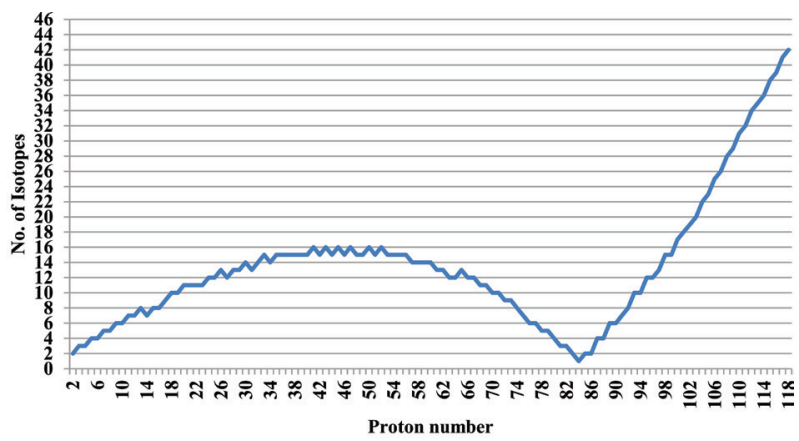

Figure 5: Estimated number of isotopes of $Z$.

Table 2: Estimated lower, mean and upper stable mass numbers of $\mathrm{Z}=2$ to 118 .

\begin{tabular}{|c|c|c|c|c|}
\hline$Z$ & $\left(A_{S}\right)_{\text {lower }}$ & $\left(A_{S}\right)_{m e a n}$ & $\left(A_{S}\right)_{\text {upper }}$ & $\begin{array}{l}\text { Estimated } \\
\text { No. of } \\
\text { Isotopes }\end{array}$ \\
\hline 2 & 4 & 4 & 5 & 2 \\
\hline 3 & 6 & 6 & 8 & 3 \\
\hline 4 & 8 & 8 & 10 & 3 \\
\hline 5 & 10 & 10 & 13 & 4 \\
\hline 6 & 12 & 12 & 15 & 4 \\
\hline 7 & 14 & 14 & 18 & 5 \\
\hline 8 & 16 & 16 & 20 & 5 \\
\hline 9 & 18 & 19 & 23 & 6 \\
\hline 10 & 20 & 21 & 25 & 6 \\
\hline 11 & 22 & 23 & 28 & 7 \\
\hline 12 & 24 & 25 & 30 & 7 \\
\hline 13 & 26 & 27 & 33 & 8 \\
\hline 14 & 29 & 29 & 35 & 7 \\
\hline 15 & 31 & 31 & 38 & 8 \\
\hline 16 & 33 & 34 & 40 & 8 \\
\hline 17 & 35 & 36 & 43 & 9 \\
\hline 18 & 37 & 38 & 46 & 10 \\
\hline 19 & 39 & 40 & 48 & 10 \\
\hline 20 & 41 & 43 & 51 & 11 \\
\hline 21 & 43 & 45 & 53 & 11 \\
\hline 22 & 46 & 47 & 56 & 11 \\
\hline 23 & 48 & 49 & 58 & 11 \\
\hline 24 & 50 & 52 & 61 & 12 \\
\hline 25 & 52 & 54 & 63 & 12 \\
\hline 26 & 54 & 56 & 66 & 13 \\
\hline 27 & 57 & 59 & 68 & 12 \\
\hline 28 & 59 & 61 & 71 & 13 \\
\hline 29 & 61 & 63 & 73 & 13 \\
\hline
\end{tabular}

\begin{tabular}{|c|c|c|c|}
\hline 30 & 63 & 66 & 76 \\
\hline 31 & 66 & 68 & 78 \\
\hline 32 & 68 & 71 & 81 \\
\hline 33 & 70 & 73 & 84 \\
\hline 34 & 73 & 75 & 86 \\
\hline 35 & 75 & 78 & 89 \\
\hline 36 & 77 & 80 & 91 \\
\hline 37 & 80 & 83 & 94 \\
\hline 38 & 82 & 85 & 96 \\
\hline 39 & 85 & 88 & 99 \\
\hline 40 & 87 & 90 & 101 \\
\hline 41 & 89 & 93 & 104 \\
\hline 42 & 92 & 95 & 106 \\
\hline 43 & 94 & 98 & 109 \\
\hline 44 & 97 & 100 & 111 \\
\hline 45 & 99 & 103 & 114 \\
\hline 46 & 102 & 106 & 116 \\
\hline 47 & 104 & 108 & 119 \\
\hline 48 & 107 & 111 & 121 \\
\hline 49 & 110 & 113 & 124 \\
\hline 50 & 112 & 116 & 127 \\
\hline 51 & 115 & 119 & 129 \\
\hline 52 & 117 & 121 & 132 \\
\hline 53 & 120 & 124 & 134 \\
\hline 54 & 123 & 127 & 137 \\
\hline 55 & 125 & 129 & 139 \\
\hline 56 & 128 & 132 & 142 \\
\hline 57 & 131 & 135 & 144 \\
\hline 58 & 134 & 138 & 147 \\
\hline 59 & 136 & 140 & 149 \\
\hline 60 & 139 & 143 & 152 \\
\hline 61 & 142 & 146 & 154 \\
\hline 62 & 145 & 149 & 157 \\
\hline 63 & 148 & 151 & 159 \\
\hline 64 & 151 & 154 & 162 \\
\hline 65 & 153 & 157 & 165 \\
\hline 66 & 156 & 160 & 167 \\
\hline 67 & 159 & 163 & 170 \\
\hline 68 & 162 & 166 & 172 \\
\hline 69 & 165 & 169 & 175 \\
\hline 70 & 168 & 171 & 177 \\
\hline 71 & 171 & 174 & 180 \\
\hline 72 & 174 & 177 & 182 \\
\hline
\end{tabular}




\begin{tabular}{llllllllll}
73 & 177 & 180 & 185 & 9 & 115 & 291 & 315 & 328 & 38 \\
74 & 180 & 183 & 187 & 8 & 116 & 294 & 318 & 332 & 39 \\
75 & 184 & 186 & 190 & 7 & 117 & 296 & 322 & 336 & 41 \\
76 & 187 & 189 & 192 & 6 & 118 & 299 & 325 & 340 & 42 \\
\hline
\end{tabular}

\section{Conclusion}

Understanding nuclear stability range in a quantum gravitational approach is challenging and most attractive issue. In this context, we tried our level best in presenting very simple and effective semi empirical formulae. The two proposed concepts, "Number of free nucleons $=A_{f}$ " and "Number of active nucleons $=A_{a}$ " can be given some consideration in understanding nuclear binding energy. The two proposed stability conditions, $\left(A_{a}-2 Z\right) \cong 0$ and $(A-2 Z) \cong(Z / 2)$ can be recommended for further investigation. By estimating the lower and upper stable mass numbers and with further study, possible number of isotopes of any proton number can be understood. With further investigation and by considering even-odd corrections, best possible range of long living super heavy elements can also be estimated.

\section{Acknowledgements}

Author Seshavatharam is indebted to professors shri M. Nagaphani Sarma, Chairman, shri K.V. Krishna Murthy, founder Chairman, Institute of Scientific Research in Vedas (I-SERVE), Hyderabad, India and Shri K.V.R.S. Murthy, former scientist IICT (CSIR), Govt. of India, Director, Research and Development, I-SERVE, for their valuable guidance and great support in developing this subject.

\section{References}

[1] K. Tennakone, Phys. Rev. D 10, 1722 (1974). https://doi.org/10.1103/physrevd.10.1722

[2] C. Sivaram and K. Sinha, Physical Review D 16, 1975 (1977). https://doi.org/10.1103/physrevd.16.1975

[3] De Sabbata V and M. Gasperini, Gen. Relat. Gravit. 10, 731 (1979). https://doi.org/10.1007/bf00756600

[4] A. Salam, C. Sivaram, Mod. Phys. Lett. A 8, 321 (1993). https://doi.org/10.1142/s0217732393000325

[5] R. Onofrio, Modern Physics Letters A 28, 1350022 (2013). https://doi.org/10.1142/s0217732313500223

[6] U. V. S. Seshavatharam and S. Lakshminarayana, Hadronic Journal 34, 379 (2011). 
[7] U. V. S. Seshavatharam, S. Lakshminarayana, Journal of Nuclear and Particle Physics 2, 132 (2012). https:// doi.org/10.5923/j.jnpp.20120206.01

[8] U. V. S. Seshavatharam and S. Lakshminarayana, Asian Journal of Research and Reviews in Physics 2, 1 (2019).

[9] U. V. S. Seshavatharam, et al., Materials Today: Proceedings 3,3976 (2016). https://doi.org/10.1016/j. matpr.2016.11.059

[10] U. V. S. Seshavatharam and S. Lakshminarayana, International Journal of Mathematics and Physics 7, 117 (2016). https://doi.org/10.26577/2218-79872016-7-1-117-130

[11] U. V. S. Seshavatharam and S. Lakshminarayana, Proceedings of the DAE-BRNS Symp. on Nucl. Phys. 61, 332 (2016)

[12] U. V. S. Seshavatharam and S. Lakshminarayana, Journal of Nuclear Physics, Material Sciences, Radiation and Applications 4, 355 (2017). https://doi. org/10.15415/jnp.2017.42031

[13] U. V. S. Seshavatharam and S. Lakshminarayana, Journal of Nuclear Sciences 4, 31 (2017).

[14] U. V. S. Seshavatharam and S. Lakshminarayana, Proceedings of the DAE Symp. on Nucl. Phys. 63, 72 (2018).

[15] U. V. S. Seshavatharam and S. Lakshminarayana, Prespacetime Journal 9, 58 (2018).

[16] U. V. S. Seshavatharam and S. Lakshminarayana, Mapana Journal of Sciences 18, 21 (2019)

[17] U. V. S. Seshavatharam and S. Lakshminarayana, J. Nucl. Phys. Mat. Sci. Rad. A. 6, 142 (2019). https:// doi.org/10.15415/jnp.2019.62024

[18] U. V. S. Seshavatharam and S. Lakshminarayana, International Journal of Innovative Studies in Sciences Engineering Technology 5, 18 (2019).
[19] U. V. S. Seshavatharam and S. Lakshminarayana, To correlate big $G$ experiments and other nuclear experiments via three atomic gravitational constants. Dec.20-21, ICAPPM-2019, Hyderabad, India. (To be appeared in IOP Journal of Physics, conference series).

[20] U. V. S. Seshavatharam and S. Lakshminarayana, Implications and Applications of Fermi Scale Quantum Gravity. (Submitted).

[21] S. Cht. Mavrodiev, M. A. Deliyergiyev, Int. J. Mod. Phys. E 27, 1850015 (2018). https://doi.org/10.1142/ S0218301318500155.

[22] X. W. Xiaa, et al., Atomic Data and Nuclear Data Tables 121-122, 1 (2018). https://doi.org/10.1016/j. adt.2017.09.001

[23] N. Ghahramany, et al., Iranian Journal of Science \& Technology A 3, 201 (2011).

[24] N. Ghahramany, et al., Journal of Theoretical and Applied Physics 6, 3 (2012).

https://doi.org/10.1186/2251-7235-6-3

[25] W. Zhang, et al., Nuclear Physics A 753, 106 (2005). https://doi.org/10.1016/j.nuclphysa.2005.02.086

[26] A. Bohr and B. R. Mottelson, Nuclear Structure Vol. 1 (W. A. Benjamin Inc., New York, Amesterdam, 1969).

[27] M. Tanabashi, et al., Phys. Rev. D 98, 030001 (2018).

[28] Helge Kragh, The Search for Super heavy Elements: Historical and Philosophical Perspectives. arXiv:1708.04064 [physics.hist-ph] (2017)

[29] Hagino K. Superheavy Elements: Beyond the 7th Period in the Periodic Table. To be published in AAPPS Bulletin. arXiv:1812.05805 [nucl-th] (2018).

[30] https://www.sciencemag.org/news/2019/01/storiedrussian-lab-trying-push-periodic-table-past-its-limitsand-uncover-exotic-new

[31] https://www.sciencenews.org/article/physics-periodictable-future-superheavy-elements 


\section{旬}

\section{Journal of Nuclear Physics, Material Sciences, Radiation and Applications}

Chitkara University, Saraswati Kendra, SCO 160-161, Sector 9-C, Chandigarh, 160009, India

Volume 7, Issue 1

August 2019

ISSN 2321-8649

Copyright: [C 2019 U. V. S. Seshavatharam and S. Lakshminarayana] This is an Open Access article published in Journal of Nuclear Physics, Material Sciences, Radiation and Applications (J. Nucl. Phy. Mat. Sci. Rad. A.) by Chitkara University Publications. It is published with a Creative Commons Attribution- CC-BY 4.0 International License. This license permits unrestricted use, distribution, and reproduction in any medium, provided the original author and source are credited. 